\title{
Spectral Energy Distributions of Fragmenting Protostellar Disks
}

\author{
Olga Zakhozhay ${ }^{1}$ and Eduard I. Vorobyov ${ }^{2,3}$ \\ ${ }^{1}$ Main Astronomical Observatory, \\ National Academy of Sciences of Ukraine, Kyiv, 03680, Ukraine \\ email: zkholga@mail.ru \\ ${ }^{2}$ Institute of Astrophysics, The University of Vienna, Vienna, 1180, Austria \\ email: eduard.vorobev@univie.ac.at \\ ${ }^{3}$ Research Institute of Physics, Southern Federal University, Rostov-on-Don, 344092, Russia
}

\begin{abstract}
In this article we show that massive and hot fragments forming in protostellar disks can leave characteristic signatures in spectral energy distributions (SEDs) of young stellar objects. This occurs when the fragment evaporates dust in its atmosphere, which results in a sharp drop in opacity and subsequent rise in the surface temperature of the fragment. These hot fragments can produce local maxima in the SEDs at 5-10 $\mu \mathrm{m}$.
\end{abstract}

Keywords. stars: formation, planetary systems: protoplanetary disks

\section{Introduction}

Observations of young protostellar disks and numerical modeling of disk formation and evolution both suggest that potostellar disks may be sufficiently massive to undergo gravitational fragmentation in the course of their evolution. Measurements of disk masses around young protostars embedded in parental cloud cores yield values that exceed $0.1 M_{\odot}$ (e.g. Jorgensen et al. 2009). Numerical hydrodynamics simulations of disk formation indicate that such massive disks are gravitationally unstable and may fragment if disk cooling is sufficiently fast (e.g. Vorobyov \& Basu 2010a). The newly-formed fragments are in the sub-stellar mass regime and are considered as embryos of giant planets and brown dwarfs which may or may not evolve into finished objects (Vorobyov \& Basu 2010b). Directly observing these embryos is however challenging because it usually requires aperture masking interferometry to distinguish between the embryo and the rest of the disk. We therefore explore possibilities of using other indirect methods for detecting fragments forming within protostellar disks.

\section{Model description}

We base our model on numerical hydrodynamics simulations of Vorobyov \& Basu (2010a), who studied the formation and evolution of protostellar disks subject to gravitational instability and fragmentation. The basic equations of hydrodynamics are solved on a polar grid in the thin-disk limit. This allows us to follow the gravitational collapse of a pre-stellar condensation (core) into the star plus disk formation stage and further to the $\mathrm{T}$ Tauri stage when most of the parental core has accreted onto the burgeoning disk. The following physical processes are taken into account: disk self-gravity via solution of the Poisson integral and disk viscosity via $\alpha$-parameterization, radiative cooling from the disk surface, stellar and background irradiation, and also viscous and shock heating. More details can be found in Vorobyov \& Basu (2010a). 


\section{Spectral energy distribution}

To construct model SEDs, we split our model young stellar object (YSO) into three constituent parts: an accreting protostar, inner disk, and outer dynamic disk. The latter include a circumstellar disk and infalling envelope (if present).

We assume that our model YSO is viewed through the outflow cavity, which allows us to use disk surface densities and temperatures directly derived from numerical hydrodynamics modeling. Below, we explain in more detail the main ideas involved in calculating the model SEDs.

a) Outer dynamic disk. We use a solution to the one-dimensional radiation transfer equation to calculate the radiative flux density $F_{\nu}$ leaving each grid cell of the disk surface

$$
F_{\nu}^{\text {disk }}=\frac{S \cos \omega}{d^{2}} B_{\nu}\left(T_{\text {eff }}\right)\left(1-e^{-\Sigma \kappa_{\nu} \sec \gamma}\right)
$$

where $B_{\nu}\left(T_{\text {eff }}\right)$ is the Plank function, $d=100 \mathrm{pc}$ is the distance to to the considered object, $\omega$ is the normal angle between a specific grid cell of the disk surface and the line of sight, $\gamma$ is the inclination angle of the disk with respect to the observer, set to zero in this article and $S$ is the physical area of a specific grid cell. The frequency-dependent opacities $\kappa_{\nu}$ are taken from Ossenkopf \& Henning (1994). The effective temperature is the maximum of irradiation and surface temperatures, which are calculated as described in
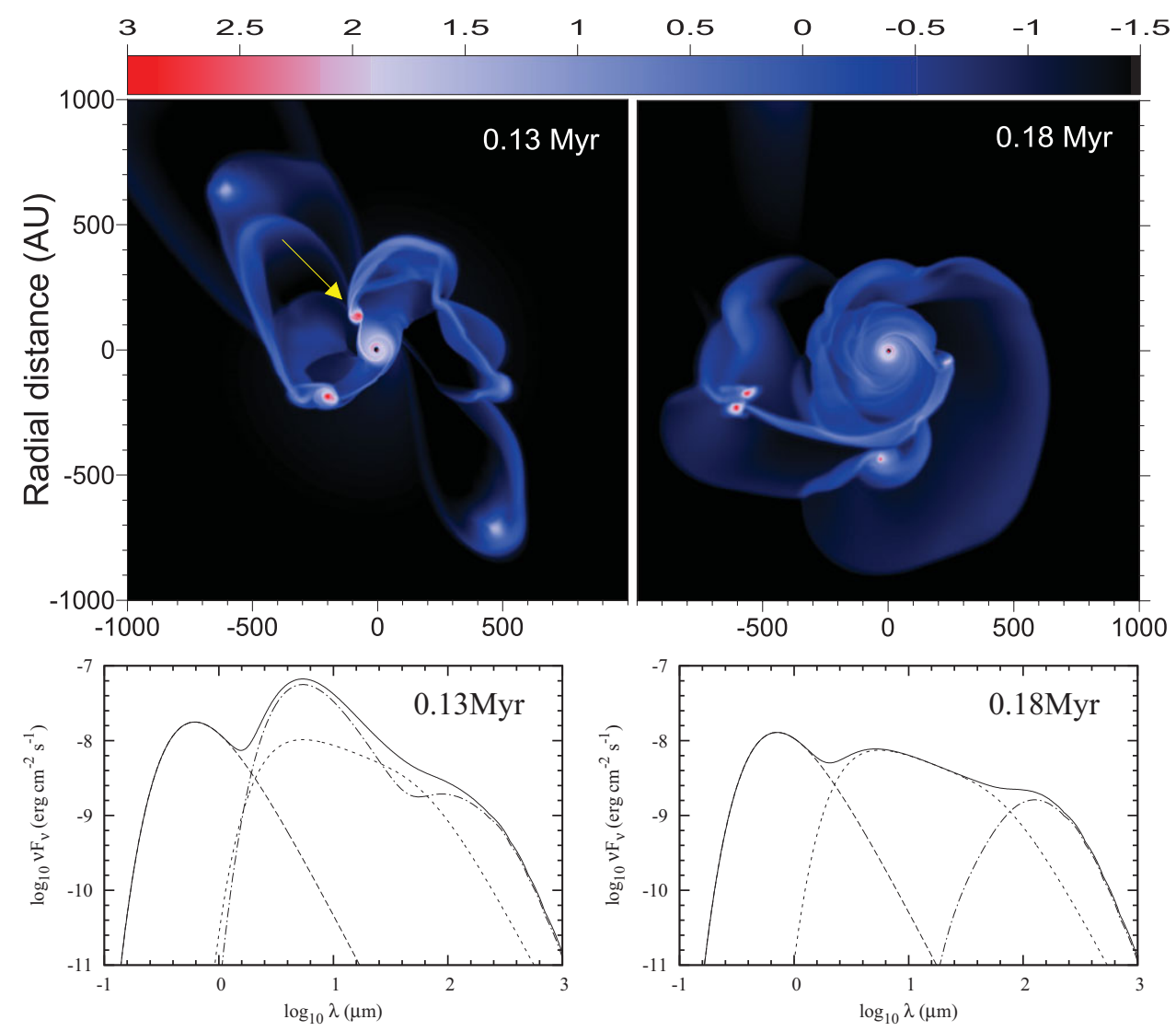

Figure 1. Top panels. Gas surface density maps of a fragmenting protostellar disk. The most massive fragment is shown by the arrow. Bottom panels. The corresponding SEDs. The total flux is shown by the solid line. 
Vorobyov \& Basu (2010a). The total flux is found by summing inputs from all $(512 \times 512)$ individual grid cells of the disk.

b) Central accreting star. The radiative flux density from the protostar $F_{\nu}^{*}$ is calculated assuming a black-body radiation spectrum with effective temperature $T_{\text {eff }}^{*}$. The latter is calculated taking into account both photospheric and accretion luminosities of the protostar.

c) The inner disk. The surface density and effective temperature of the inner disk are calculated by extrapolating the corresponding azimuthally-averaged profiles of the outer dynamic disk down to the dust sublimation radius $R_{\text {d.s }}$. The latter is calculated assuming the dust evaporation temperature of $1500 \mathrm{~K}$ (Dunham et al. 2010). The resulting profiles are then substituted into equation (3.1) to obtain the input from the inner disk to the total SED.

\section{Results}

The top panels in Figure 1 show the gas surface density maps of a protostellar disk formed as a result of gravitational collapse of a cloud core with initial mass $M_{\mathrm{c}}=1.2 M_{\odot}$. Only the inner $2000 \times 2000$ AU box is shown, the total computational area is ten times greater. The time (in Myr) is counted since the formation of the protostar. Disk fragmentation is evident in both panels. The masses and effective temperatures of the fragments are however different. The most massive and hot fragment shown with the arrow in the top-left panel of Fig. 1 has mass and effective temperature $0.06 M_{\odot}$ and $730 \mathrm{~K}$, respectively. The other fragment in the same panel is considerably lighter and cooler, with mass and temperature equal to $0.03 M_{\odot}$ and $74 \mathrm{~K}$, respectively. After $0.05 \mathrm{Myr}$ of evolution (top-right panel), the most massive fragment has migrated onto the star due to angular momentum exchange with spiral arms (Vorobyov \& Basu 2010a) and the remaining fragments have masses and temperatures not exceeding $0.03 M_{\odot}$ and $70 \mathrm{~K}$ respectively.

This change in the fragment properties is reflected in the corresponding SEDs presented in the bottom panels of Figure 1. Dashed, dotted, and dash-dotted lines show individual contributions from the protostar, inner disk, and outer dynamic disk, respectively, while the solid line is the total flux. The SED at $t=0.13 \mathrm{Myr}$ shows a well-defined peak at $\approx 5 \mu \mathrm{m}$, whereas the SED at $t=0.18 \mathrm{Myr}$ lacks such a feature. The occurrence of a peak at 5-10 $\mathrm{mm}$ may thus reflect the presence of a massive and hot fragment in the disk. The surface temperature of the fragment should exceed several hundred Kelvin. This can happen if the gas temperature in the fragment interiors exceeds the dust sublimation temperature and the opacity of the fragment drops considerably. Such fragments have not yet dissociated molecular hydrogen and therefore have not yet collapsed to a sub-stellar/planetary size, but have already evaporated dust in the atmosphere, thus revealing themselves as hot proto-brown dwarf embryos.

Acknowledgements. E.I.V. acknowledges support from the RFBR grant 11-02-92601KO. O.Z. acknowledges travel support from the IAU.

\section{References}

Dunham, M. M., Evans, N. J., II, Terebey, S., Dullemond, C. P., \& Young, C. H. 2010, ApJ, 710,470

Jorgensen, J. K., van Dishoeck, E. F., Visser, R., Bourke, T. L., Wilner, D. J., Lommen, D., Hogerheijde, M. R., \& Myers, P. C. 2009, A\&̈A, 507, 861

Ossenkopf, V. \& Henning, Th. 1994, A\&A, 291, 943

Vorobyov, E. I. \& Basu, S. 2010, ApJ, 719, 1896

Vorobyov, E. I. \& Basu, S. 2010, ApJL, 714, 133 